Reprod. Nutr. Dévelop. 1980, 20 (4 A), 1111-1123.

\title{
Effets du plasma séminal sur la survie et la fertilité des spermatozoïdes conservés in vitro
}

\author{
par J. M. CORTEEL
}

Stafion de Physiologie de la Reproduction, I.N.R.A., Nouzilly 37380 Monnaie, France.

Summary. Effects of seminal plasma on the survival and fertility of spermatozoa kept in vitro.

In sperm cell populations kept in vitro, the profiles of motile cell number and motility are always influenced by the seminal plasma. The non-epididymal fraction of the seminal plasma first enhanced and then depressed bull, ram and goat sperm motility or immediately depressed that of horses and rabbits. The secretions of Cowper's gland (male goat) or of the seminal vesicles (boar) were implicated in the depressive effect of the non-epididymal plasma fraction on the maintenance of sperm motility. These secretions were responsible for variations in motility correlated with variations in sperm fertility. The level and significance of these correlations indicated that the seminal plasma might be involved in sperm fertility, perhaps owing to a type A lecithinase secreted by Cowper's gland (bull, ram and male goat) or by a protein of vesicular origin (boar).

\section{Introduction.}

Chez les mammifères supérieurs, le plasma séminal résulte du mélange, au moment de l'éjaculation, des secrétions épididymaires contenant les spermatozoïdes avec celles des autres glandes du tractus génital : les glandes annexes. Ainsi, le plasma séminal sera défini comme tout ce qui, dans l'éjaculat, n'est pas spermatozoïdes morts ou vivants.

Ce milieu a été l'objet de très nombreuses investigations biochimiques mais en dépit de remarquables synthèses des connaissances qui en ont découlé (Mann, 1964), le rôle du plasma séminal dans la physiologie des spermatozoïdes éjaculés reste encore très obscur. Ceci est probablement dû à ce que toutes les études ont été faites in vitro dans des conditions qui ne reproduisent que très imparfaitement celles qui existent in vivo.

Les modifications apportées in vitro à l'environnement normal des spermatozoïdes (exposition à la lumière, diminution ou augmentation $\mathrm{du} \mathrm{pH}$, variations thermiques, apports de nutriments ou de protecteurs, etc...) entrainent des variations du métabolisme et/ou de la survie des spermatozoïdes. De plus, les mécanismes d'élimination des spermatozoïdes morts qui fonctionnent in vivo (phagocytoses, excrétion urinaire, 
résorption, etc...) n'existent pas in vitro où les spermatozoïdes vivants sont contraints de vivre avec les spermatozoïdes morts et les produits de leur décomposition.

En dépit des modifications apportées à l'environnement des spermatozoïdes, on esł parvenu à créer des systèmes qui parviennent à maintenir non seulement la vie apparente du spermatozoïde mais aussi sa fertilité, c'est-à-dire, son aptitude à féconder un œuf dont le développement aboutit à la naissance d'un jeune viable. Ainsi, des millions de mammifères naissent chaque année d'insémination artificielle. Cette remarquable performance a été accomplie avant que l'on ait su relier avec précision les événements mesurables sur le sperme in vitro et la fertilité des spermatozoïdes.

De nouvelles conditions d'observation de la survie des spermatozoïdes font apparaître dans des conditions également nouvelles de protection, des relations étroites entre leur survie, leur motilité ef leur fertilité. On commence aussi à mieux connaître les effets du plasma séminal sur les paramètres de survie in vitro des spermatozoïdes qui rendent compte, en partie au moins, de leur fertilité. Il est donc intéressant de s'interroger une nouvelle fois sur le rôle éventuel du plasma séminal dans la fertilité des spermatozoïdes conservés in vitro.

\section{1. - Plasma séminal et survie in vitro des spermatozoïdes.}

Les paramètres les plus usités pour mesurer la survie in vitro des spermatozoïdes sont, d'une part, l'évolution quantitative de la population de cellules estimées mobiles en fonction du temps de conservation, d'autre part, le devenir de la motilité ou vitesse de déplacement des spermatozoïdes dans le même temps. La première estimation est faite tout au plus quelques heures après l'éjaculation.

\section{Effets du plasma séminal pris dans son ensemble.}

Pendant longtemps, le plasma séminal a éfé considéré comme indispensable à la survie des spermatozoïdes. Cette croyance découlait d'observations montrant que des spermatozoïdes mouraient, notamment ceux de faureau, lorsque la concentration du milieu de conservation en plasma séminal était réduite à l'extrême par une dilution excessive du sperme ou lavage des spermatozoïdes (Mann, 1964).

A l'opposé, Dott, Harrison et Foster (1979) ont montré que la motilité ef la survie des spermatozoïdes éjaculés de taureau, de bélier et de lapin n'étaient pas inhibées après que le sperme ait élé dilué $6000 \mathrm{fcis}$. Ceci est également vrai pour les spermatozoïdes du sperme épididymaire des mâles des mêmes espèces dilué 20000 fois. Des taux appréciables de plasma séminal ne sont donc pas indispensables dans le milieu de conservation des spermatozoïdes pour les maintenir en vie.

Au contraire, des travaux de plus en plus nombreux montrent que le plasma séminal diminue globalement le maintien de la motilité et/ou de la survie in vitro des spermátozoïdes de taureau (Shannon, 1965 ; Bennet et Dott, 1966 ; Dott, 1974 ; Dott, Harrison et Foster, 1979), de bélier et de lapin (Dott, Harrison et Foster, 1979) ef d'étalon (Werthessen, 1956 ; Werthessen ef al., 1957 ; Haag, 1959 ; Langlois, comm. pers. 1978). Nous avons nous-même montré que la suppression d'une grande proportion du plasma séminal par lavage des spermatozoïdes de bouc dès la récolte améliore 
leur survie après refroidissement à $+4^{\circ} \mathrm{C}$, congélation, dégel et au-delà (Corteel, 1974). En outre, dans cette espèce, les spermatozoïdes congelés avec leur plasma séminal et potentiellement réanimables au dégel quelques jours après congélation perdent progressivement cette potentialité après deux mois de séjour dans l'azole liquide (Corteel, 1975). Ces faits nous ont paru moins surprenants lorsque nous avons constaté que 50 p. 100 des spermatozoïdes ainsi congelés sont porteurs d'altérations membranaires importantes après refroidissement à $+4^{\circ} \mathrm{C}$, avant même d'être congelés (ruptures et vésiculisations membranaires avec présence de globules à la périphérie de la rupture ou de la vésiculisation) (Courtens et Corteel, non publié ; tabl. 1). Des spermatozoïdes fragilisés à ce point pourraient avoir subi la congélation avec un succès tout relatif et se rompre à l'occasion des moindres mouvements internes du congelat.

\section{TABLEAU 1}

Taux de spermatozoïdes avec ruptures ou vésiculisations membranaires. Espèce caprine (Courtens et Corteel, non publié)

— Spermatozoïdes éjaculés non lavés de mâles entiers

- Spermatozoïdes éjaculés lavés de mâles entiers

- Spermatozoïdes éjaculés non lavés de mâles cowperectomisés ..

- Spermatozoïdes éjaculés lavés de mâles cowperectomisés .....

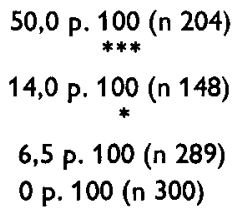

N. A.

Le milieu de dilution et de congélation est le même dans les 4 cas envisagés.

$$
\begin{aligned}
& * * *=\mathrm{P}<0,001 . \\
& *=\mathrm{P}<0,05 . \\
& \text { N. A. }=\text { Non analysé. }
\end{aligned}
$$

$2^{\circ}$ Effets parcellaires du plasma séminal.

a) Effet du plasma non épididymaire. - L'amplitude et la longévité de la motilité des spermatozoïdes épididymaires demeurés dans leur plasma d'origine sont diminuées par l'adjonction de plasma séminal au milieu de conservation. Ceci a été montré pour les spermatozoìdes de taureau (Shannon, 1965 ; tabl. 2) mais les travaux de Dott, Harrison et Foster (1979) apportent une précision supplémentaire : la diminution de la motilité fait suite à une stimulation de celle-ci en début de conservation

\section{TABLEAU 2}

Temps nécessaires à l'inhibition de la survie des spermatozoïdes de taureau conservés $24 h$ à tempérafure ambiante puis incubés d̀ +37 oC

- Plasma épididymaire + spermatozoïdes $=24 \mathrm{~h}$

- Plasma éjaculé + spermatozoïdes $=4 \mathrm{~h}$

(Shannon, 1965) 
comme chez le bélier (fig.1) et le bouc (Nunes-Ferreira, Baril ef Corteel, résultats non publiés).
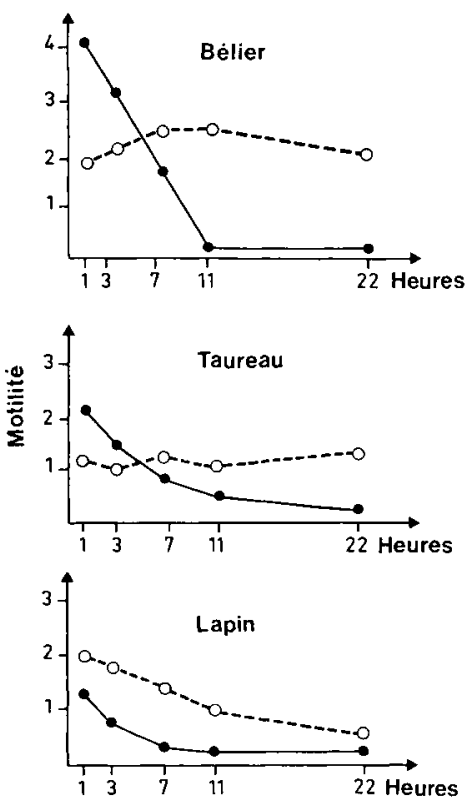

FIG. 1. - Devenir de la motilité des spermatozoìdes épididy maires conservés à $+30^{\circ} \mathrm{C}$ avec (•-—) ou sans plasma séminal (o-o) (Dott, Harrison et Foster, 1979).

FIG. 2. - Effet de l'origine du sperme sur la survie des spermafozoides de bouc après refroidissement da $+4^{\circ} C(A)$, congélotion ef décongélation (B) ef 2 h d'incubation à $+37^{\circ} \mathrm{C}$ (C) (Chemineau, 1978).

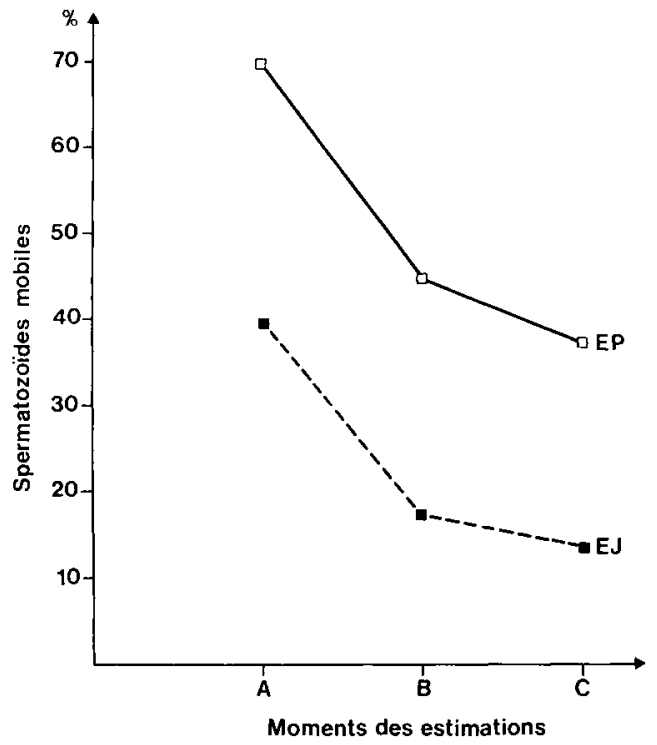

EP: Sperme de la queue de l'épididyme

EJ : Sperme èjaculé 
Chez le lapin (flg. 1) et l'étalon, la diminution de la motilité par le plasma séminal apparaît dès le début de la conservation.

Chez le bouc, le pourcentage de spermatozoïdes épididymaires mobiles et leur motilité après refroidissement à $+4^{\circ} \mathrm{C}$, congélation, dégel ef incubation à $+37^{\circ} \mathrm{C}$ sont toujours d'un niveau supérieur à celui des spermatozoïdes éjaculés (Haller, Chemineau et Corteel, $1978:$ fig. 2 et 3).

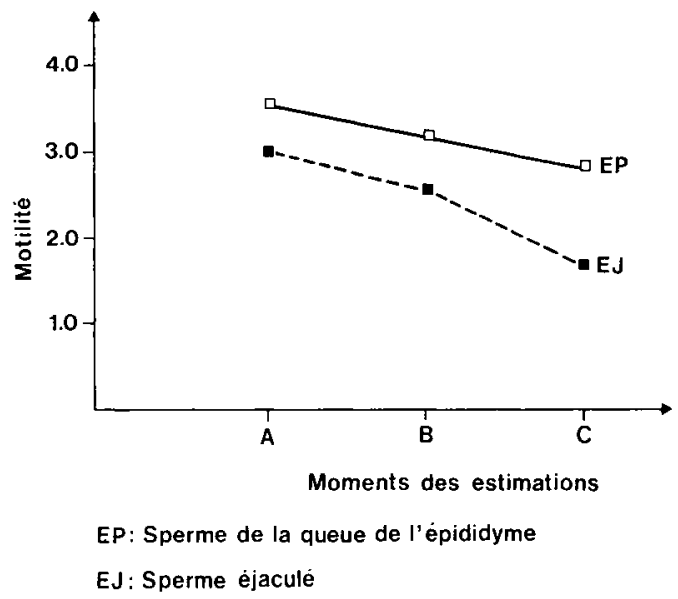

FIG. 3. - Effet de l'origine du sperme sur la motilité des spermatozoïdes de bouc, après refroidissement $\grave{d}+4^{\circ} \mathrm{C}(\mathrm{A})$, congélation ef décongélation (B) et $2 \mathrm{~h}$ d'incubation d̀ $+37^{\circ} \mathrm{C}$ (Haller et al., 1978).

La motilité et le pourcentage de spermatozoïdes mobiles du sperme épididymaire de verrat exposés brutalement à une température égale ou inférieure à $0^{\circ} \mathrm{C}$ sont toujours supérieurs à ceux des mêmes spermatozoïdes ayant reçu une adjonction de plasma séminal (Lasley et Bogart, 1944 ; Pursel, Johnson et Rampacek, 1972).

Chez le bouc, le taux de diminution de la motilité induit par le plasma séminal entier après congélation et dégel est très supérieur à celui induit par le plasma épididymaire (Haller, Chemineau et Corteel, 1978 : tabl. 3). Il est variable avec les saisons de récolte du sperme, les boucs ef les éjaculats.

\section{TABLEAU 3}

Taux (p. 100) de diminution de la motilité induits par le plasma épididymaire ou séminal par la congélation puis par une incubation de $2 \mathrm{~h} d+37^{\circ} \mathrm{C}$ après décongélation (espèce caprine)

\begin{tabular}{cccc}
\hline Origine du sperme & Congélation & & Incubation \\
\hline Queve de l'épididyme $\ldots .$. & 10 & $*$ & 12 \\
& $15 * *$ & & $* * *$ \\
Ejaculat ............... & 15 & $* * *$ & 34 \\
\hline
\end{tabular}

* $\quad P<0,05$.

$* * * P<0,01$. 
Le plasma épididymaire seul est, soit plus favorable, soit moins défavorable à la survie in vitro des spermatozoïdes que le plasma séminal entier. On peut donc penser que la fraction non épididymaire du plasma séminal contient plus d'éléments défavorables à la survie des spermatozoïdes que le plasma épididymaire.

b) Effets des excrétats des spermatozoïdes. - Le plasma éjaculé contient des cytochromes et leur équipement enzymatique, de l'hyaluronidase, de l'acrosine, des lipoprotéines, des protéines intra-cellulaires, etc... qui peuvent se détacher des spermatozoïdes simplement endommagés ou diffuser des spermatozoïdes morts (Mann 1964). Les effets de la plupart de ces substances sur la survie in vitro des spermatozoïdes sont peu connus à l'exception de ceux des oxydases dans le sperme de bovin.

Tosic $(1947)$ et Tosic et Walton $(1946,1950)$ ont montré que les spermatozoïdes de taureau étaient capables d'oxyder les acides aminés aromatiques. Cette oxydation produit des peroxides qui inhibent la respiration, la survie ef la motilité des spermatozoïdes. Les possibilités d'oxydation sont davantage liées à la présence de spermatozoïdes morts qu'à celle des spermatozoïdes vivants (Shannon et Curson, 1972). Les conséquences de ces oxydations (diminution de la respiration, de la survie et de la motilité) sonf observées après exposition du sperme à la lumière du jour (Norman et al., 1959). Chez le lapin, le lavage des spermatozoïdes exposés à la lumière du jour stimule leur respiration (Hamner et Williams, 1961). Les oxydases peuvent être ceux des cytochromes, pigments intracellulaires capables d'absorber l'énergie lumineuse et de la transférer à une protéine cellulaire ou extracellulaire qui s'oxyde avec

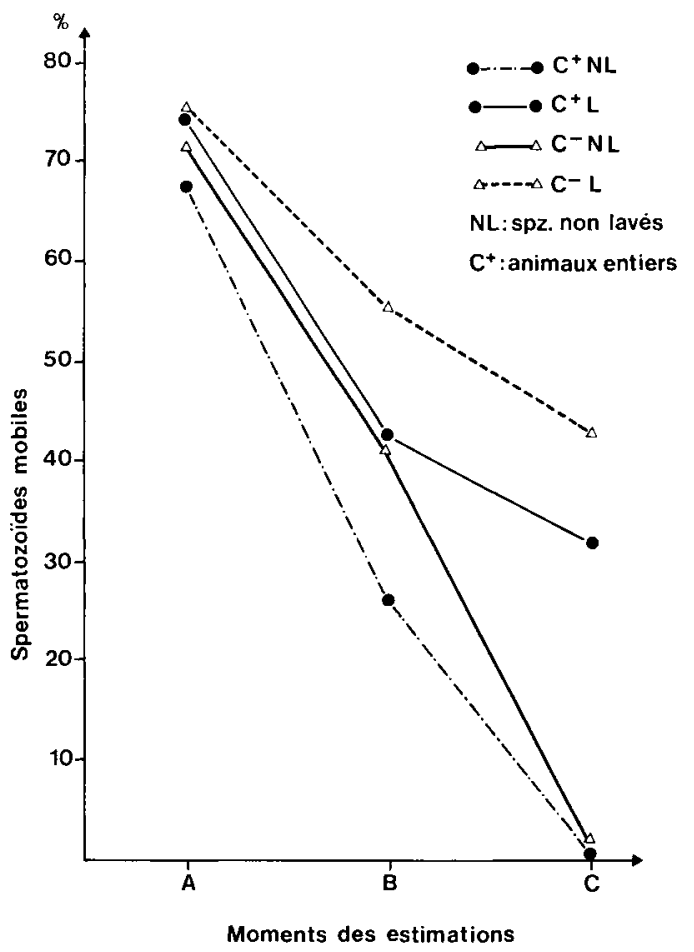

FIG. 4. - Effets de la "cowperectomie " $\left(C^{-}\right)$et du lavage des spermatozoïdes de bouc à la récolte (L). sur la survie des spermatozoïdes après refroidissement $\grave{a}+4^{\circ} \mathrm{C}(\mathrm{A})$, congélation ef décongélation (B) ef $2 h$ d'incubation à $+37^{\circ} \mathrm{C}$ (C) (Esteva-Hernandez, 1976). 
libération de peroxydes. Cet effet nuisible de l'oxydation photochimique est aggravé par l'adjonction de jaune d'œuf au milieu de conservation et par le fait que le sperme des mammifères est dépourvu de catalase (Mann, 1964) qui détruit les peroxydes. L'EDTA protège les spermatozoïdes de leurs effets nocifs (Salisbury, Van Demark et Lodge, 1978).

c) Effet de la suppression d'une ou plusieurs glandes annexes. - Les spermatozoïdes de verrats vésiculectomisés libèrent moins de GOT et de lactate déshydrogénase que ceux des verrats entiers surtout après avoir séjourné à des températures inférieures à $15^{\circ} \mathrm{C}$ (Moore, Hall et Hibbitt, 1976).

Chez le bouc, la cowperectomie entraine une amélioration de la survie des spermatozoïdes refroidis à $+4^{\circ} \mathrm{C}$ puis incubés à $+37^{\circ} \mathrm{C}$ après décongélation et une réduction très sensible du taux de spermatozoïdes porteurs d'anomalies membranaires (6,5 p. 100 chez les animaux cowperectomisés contre 50 p. 100 chez les animaux entiers : Courtens et Corteel : tabl. 1). Le lavage des spermatozoïdes des boucs cowperectomisés ne supprime pas totalement les anomalies membranaires. Ceci est vraisemblablement lié au fait que la cowperectomie laisse subsister dans le muscle bulbo-uréthral quelques ilôts du tissu secrétoire caractéristique des glandes de Cowper (Locatelli et al., 1978). Le lavage des spermatozoïdes éjaculés issus de boucs cowperectomisés leur permet après refroidissement $\grave{a}+4^{\circ} \mathrm{C}$ puis $\grave{a}-196^{\circ} \mathrm{C}$ de mieux survivre que les spermatozoïdes non lavés des mêmes animaux (Esteva-Hernandez, 1976 : fig. 4 et 5). Les secrétions des glandes de Cowper paraissent donc avoir un rôle déterminant dans la variation de la motilité des spermatozoïdes de bouc après congélation.

FIG. 5. - Effets de la « cowperectomie $\gg\left(\mathrm{C}^{-}\right)$et du lavage des spermatozoïdes de bouc à la récolfe $(\mathrm{L})$ sur la motilité des spermatozoïdes après refroidissement d̀ +4 o C (A), congélation ef décongélafion (B) et $2 h$ d'incubation à $+37^{\circ} \mathrm{C}$ (C) (Esteva-Hernandez, 1976).

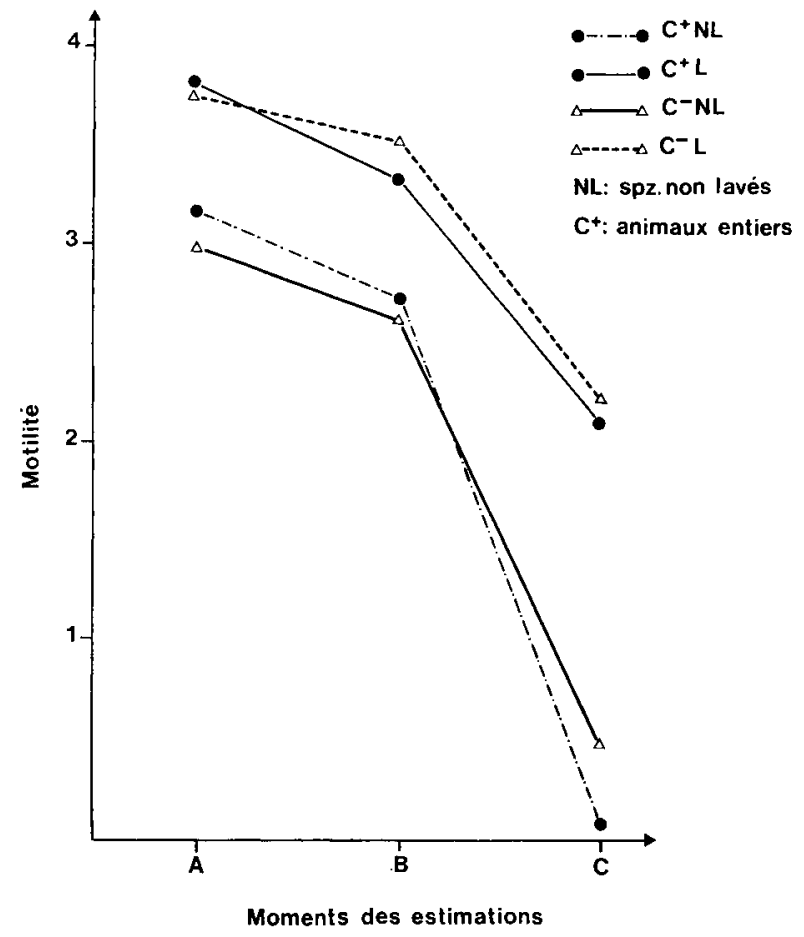




\section{II. - Conséquences de la modulation de la survie in vitro des sper matozoïdes par le plasma séminal sur la fertilité des spermatozoïdes.}

De nombreux auteurs ont essayé de relier les paramètres de survie in vitro et la fertilité des spermatozoïdes (Salisbury, Van Demark et Lodge, 1978). La plupart des corrélations trouvées sont toujours de faible niveau, peut-être en raison de conditions trop ou trop peu sévères auxquelles étaient soumis les spermatozoìdes. Cependant, la survie et la motilité des spermatozoïdes en cours d'incubation après congélation et dégel font apparaître chez le taureau des liaisons statistiquement significatives avec la fertilité : lorsque le sperme a été incubé pendant $3 \mathrm{~h}$, la fertilité après insémination est corrélée avec le pourcentage de spermatozoïdes estimés mobiles $(r=0,53)$ ef avec leur motilité $(r=0,74)$. Dans ce dernier cas, les variations de motilité expliquent 50 p. 100 de la variance de la fertilité (Pelot et al., 1976).

Chez le bouc, lorsqu'on mesure les variations de motilité des spermatozoïdes de saison sexuelle au cours d'une incubation de $2 \mathrm{~h}$ après décongélation, la fertilité est corrélée positivement avec la diminution de motilité pour des pertes de motilité comprises entre 20 p. 100 et 50 p. 100 . Cette corrélation s'inverse au-delà de 50 p. 100 : Corteel ef al., 1980, fig. 6).

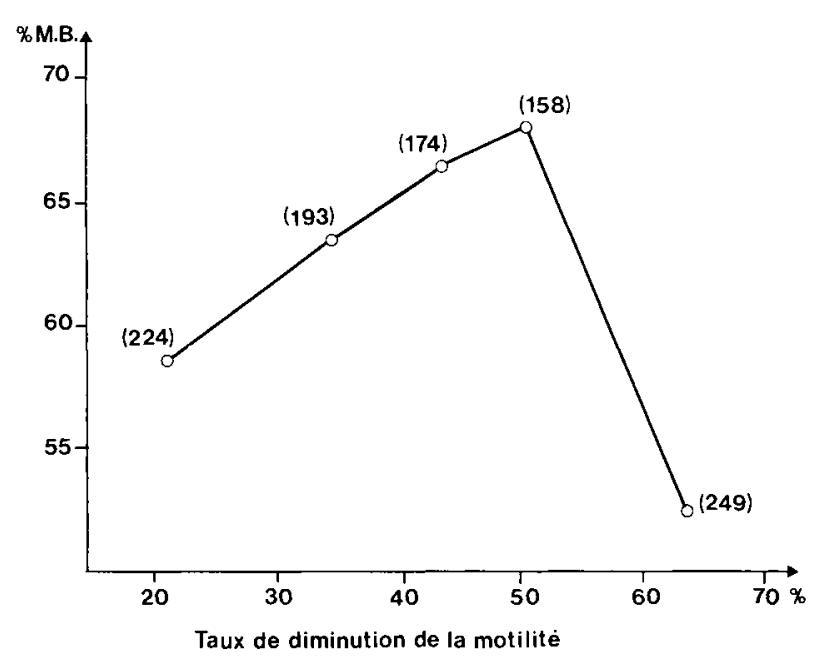

(): nombre de femelles insèminèes

FIG. 6. - Fertilité des éjoculats de boucs (p. 100 M. B.) en fonction du taux de diminution de la motilité des spermatozoides au cours d'une incubation de $2 \mathrm{~h}$ d̀ $+37^{\circ} \mathrm{C}$ après congélation ef décongélation (Corteel et al., 1980).

Chez les porcins, le taux de truies fécondées par des spermatozoïdes éjaculés congelés puis décongelés de mâles entiers a été de 28 p. 100 ( 32 femelles) contre 36 p. 100 (31 femelles) pour ceux issus de mâles vésiculectomisés (Moore et Hibbitt, 1977). Cette différence n'est pas significative mais le bas niveau moyen de l'essai semble 
indiquer que les conditions de la congélation du sperme et de l'insémination des truies ne sont pas satisfaisantes. On peut cependant se demander si la tendance favorable liée à la vésiculectomie ne reflète pas une différence plus importante qui aurait été « écrasée » par des facteurs limitants d'ordre technologique et/ou physiologique. En effet, il est possible d'obtenir 58 p. 100 de mise-bas (382 femelles) après insémination de spermatozoïdes congelés-décongelés de mâles entiers, au cours d'un seul œstrus, et ce taux peut même atteindre 64,5 p. $100(n=282)$ pour des truies multipares présentant les éléments du comportement d'œstrus qui permettent la fertilité optimale de la femelle après insémination artificielle (Paquignon, 1979, comm. pers.).

\section{Discussion.}

Le plasma séminal stimule la motilité des spermatozoïdes de taureau et de bélier à $+30^{\circ} \mathrm{C}$ et de bouc à $+37^{\circ} \mathrm{C}$ mais la cessałion complète de cette stimulation est d'autant plus rapide que son amplitude initiale est plus forte. Elle est très rapide chez le bouc ( 3 hrs environ), plus lente chez le bélier ( $11 \mathrm{hrs}$ ) ef beaucoup plus lente chez le taureau (15 h environ). Dès la $5^{\mathrm{e}}$ heure de conservation, la motilité des spermatozoïdes épididymaires non exposés au plasma séminal est supérieure à celle de leurs homologues exposés au plasma séminal. Elle se maintient à un niveau correct pendant $22 \mathrm{~h}$ chez le taureau et le bélier. Entre ces trois espèces, la différence d'intentensité et de persistance de l'effet stimulant du plasma séminal induit des différences de précocité, d'amplitude et de durée de son effet dépressif sur le maintien de la motilité en cours de conservation.

Chez le lapin et l'étalon, il est immédiat. Chez le verrat, il est perceptible après que les spermatozoïdes aient séjourné à $+15^{\circ} \mathrm{C}$ à en juger par les pertes enzymatiques (Moore, Hall et Hibbitt, 1976). Il est exacerbé par la congélation, un séjour prolongé à $-196^{\circ} \mathrm{C}$ ef l'incubation qui suit la décongélation des spermatozoïdes de bouc. C'est aussi pour cette raison que les techniques de congélation des spermatozoïdes de verrat éliminent le plasma séminal (Paquignon et al., 1978). Il est donc difficile de contester l'effet dépressif du plasma séminal sur la survie in vitro des spermatozoïdes. Cependant, qu'on les estime en phase de stimulation ou d'inhibition, le pourcentage de spermatozoïdes mobiles et la motilité sont toujours modulés par le plasma séminal. Dans des conditions précises, la variation de ces paramètres explique une large part de la variation de la fertilité des populations de spermatozoïdes concernées : motilité des spermatozoïdes de taureau après $3 \mathrm{~h}$ d'incubation suivant la décongélation ; évolution de la motilité dans des conditions voisines chez le bouc.

La relation avec la fertilité est en général moins étroite avec le pourcentage de spermatozoïdes mobiles qu'avec la motilité. Le pourcentage de spermatozoïdes mobiles reflète davantage le taux de spermatozoïdes encore vivants alors que la motilité renseigne sur le degré de stabilité de ces spermatozoïdes.

L'origine anatomique du plasma non épididymaire concernée dans la modulation de la survie « in vitro » des spermatozoïdes varie selon les espèces : secrétions des vésicules séminales chez le verrat, secrétions des glandes de Cowper chez le bouc, mais jusqu'à présent les mécanismes en jeu restent du domaine des hypothèses. 
Les glandes de Cowper du taureau, du bélier et du bouc secrètent une lécithinase (Iritani et Nishikawa, 1964) à une concentration telle chez le bouc qu'elle permet I'hydrolyse des lécithines du jaune d'œuf avec libération importante d'acides gras ef de lysolécithine (Roy, 1957). Il est possible qu'elle soit capable d'hydrolyser aussi les phospholipides présents dans la membrane des spermatozoïdes.

Selon sa concentration dans le milieu, la lysolécithine peut avoir les effets les plus divers sur les spermatozoïdes :

- Mort immédiate des spermatozoïdes de verrat ef de bouc introduits dans une dilution au 1/1 000 (Aamdal, Lyngset et Fossum, 1965).

- Dispersion complète des contenus acrosomiques des spermatozoïdes de verrat exposés $2 \mathrm{~h}$ à une concentration de $496 \mu \mathrm{g} / \mathrm{ml}$ (Jones, 1976).

- Suppression de la membrane plasmique et endommagement des mitochondries des mêmes spermatozoïdes soumis à la même concentration mais seulement pendant 10 sec. (Jones, 1976).

- Suppression de la membrane plasmique ef ruptures cellulaires dans une faible proportion de spermatozoïdes de verrat exposés $10 \mathrm{sec}$ à la concentration de $49,6 \mu \mathrm{g} / \mathrm{ml}$ (Jones, 1976).

- Inhibition de la respiration des spermatozoïdes de bélier par 0,5 nmole (Hartree ef Mann, 1960).

- Stimulation de la respiration des spermatozoīdes de bélier par 0,04 nmole (Hartree et Mann, 1960).

Par ailleurs, il a été montré dans de nombreuses occasions que la lysolécithine peut être impliquée dans les fusions membranaires (Poole, Howell et Lucy, 1970). Elle peut aussi après recyclage redonner la lécithine dont elle est issue (Mulder, Van den Berg et Van Deenen, 1965).

Chez les bovins, les ovins ef les caprins, la stimulation de la motilité par le plasma séminal en début de conservation pourrait résulter d'une concentration faible mais croissante de lysolécithine liée à l'hydrolyse naissante puis croissante des phospholipides du spermatozoïde. In vivo, cette stimulation faciliterait la progression des spermatozoïdes au travers et au-delà du cervix. Passé le seuil de concentration favorable à la respiration et à la motilité, la déstabilisation des membranes se poursuivrait jusqu'à s'étendre à une large fraction de la population concernée. Cette déstabilisation pourrait s'opérer pendant la traversée de l'utérus par des spermatozoïdes respirant peu. La diminution de la concentration des secrétions utérines en spermatozoïdes à l'approche de la jonction utéro-tubaire permettrait un retour à des concentrations favorables à la respiration et à la motilité des spermatozoïdes pour le franchissement de la jonction utéro-tubaire et à l'accolement avec l'ovocyłe. Un nouvel accroissement de la concentration en lysolécithine serait alors nécessaire pour détruire les annexes non nucléaires du spermatozoïde. La possibilité de régénération des lécithines ne s'oppose encore pas à une telle hypothèse.

In vitro, compte-tenu de l'activité lécithinasique très élevée des secrétions des glandes de Cowper du bouc, on peut imaginer que le seuil de toxicité de la lysolécithine soit rapidement atteint par les spermatozoïdes de l'espèce, conservés en présence de plasma séminal à des concentrations élevées $\left(400\right.$ à $500 \times 10^{6} \mathrm{spz}$. $\left./ \mathrm{ml}\right)$. Le lavage des spermatozoïdes dès la récolte permettrait de limiter l'effet de la lécithinase à la 
déstabilisation des membranes. Toufefois, cette déstabilisation ne devrait pas être trop avancée comme le suggère, entre autres possibilités, la liaison entre la diminution de la motilité des spermatozoïdes après décongélation ef l'augmentation de leur fertilité mais à laquelle fait suite une chute dramatique de la fertilité.

Chez le taureau, l'ensemble des opérations techniques de dilution, de refroidissement, de congélation, de décongélation et d'incubation s'effectue tandis que la motilité des spermatozoïdes est stimulée par le plasma séminal, il n'est donc pas surprenant que la meilleure fertilité soit associée à la meilleure motilité. S'il y a production de lysolécithine son taux devrait demeurer faible pendant longtemps, compte-tenu de la très faible sécrétion de lécithinase par les glandes de Cowper de taureau (Iritani et Nishikawa, 1964).

Les concentrations très élevées (bouc) et élevées (bélier) en lécithinase expliqueraient alors les difficultés rencontrées pour congeler les spermatozoïdes dans ces espèces. De tous les cas, la congélation des spermatozoïdes de bouc n'a été couronnée de succès qu'après élimination de la plupart du plasma séminal par lavage des spermatozoïdes dès la récolte.

\section{Conclusion.}

Le plasma séminal induit des variations importantes du pourcentage de spermatozoïdes mobiles ef de la motilité dans le sens d'une augmentation puis d'une diminution chez le taureau, le bélier et le bouc, toujours dans le sens d'une diminution dans les autres espèces étudiées (cheval, lapin).

II a été possible d'expliquer jusqu'à 50 p. 100 de la variance de la fertilité après insémination par les variations de motilité des spermatozoïdes de taureau. Cette liaison prend toute son importance lorsqu'on considère qu'elle n'intègre pas les sources de variation de la fertilité liées aux interactions entre les spermatozoïdes et les secrétions du tractus génital de la femelle. Dans ces conditions, il est difficile d'exclure une participation du plasma séminal dans la fertilité des spermatozoïdes conservés in vitro.

Chez les bovins, les spermatozoïdes les plus fertiles seraient ceux qui auraient été le mieux stimulés par le plasma séminal. Chez les caprins, un minimum d'effet dépressif du plasma séminal sur la motilité paraît indispensable à la meilleure fertilité des spermatozoïdes. Cet effet minimum est obtenu en réduisant considérablement la concentration de plasma séminal dans le milieu de conservation. C'est également en supprimant le plasma séminal que l'on parvient le plus aisément à congeler les spermatozoides de verrat.

Chez les bovins, ovins et caprins, les variations de concentration en lécithinase des sécrétions des glandes de Cowper pourraient expliquer la plus ou moins grande facilité avec laquelle on peut congeler les spermatozoïdes en raison des conséquences opposées que cet enzyme peuf avoir suivant sa concentration.

Chez le verrat, c'est apparemment une protéine d'origine vésiculaire qui diminuerait la fertilité des spermatozoïdes congelés, décongelés.

Les travaux à venir infirmeront ou confirmeront les hypothèses avancées et, par 
delà les substances plasmatiques concernées dans la survie et la fertilité des spermatozoïdes de mammifères conservés in vitro, il serait intéressant de connaître l'origine des variations de la sensibilité des spermatozoïdes au plasma séminal.

\author{
Symposium sur "Les glandes annexes mâles ». \\ 3 e Réunion franco-britannique des Sociétés d'Ełude \\ de la Fertilité et de la Stérilité, \\ Gaillon, 14-16 décembre 1979.
}

\title{
Références
}

AAMDAL J., LYNGSET O., FOSSUM K., 1965. Toxic effect of lysolecithin on sperm. A preliminary report. Nord. vet. Med., 17, 633-634.

BENNETT J. P., DOTT H. M., 1966. An effect of bovine seminal plasma on the impedance change frequency of epididymal spermatozoa collected from the living bull. J. Reprod. Fert., 12, 327336.

CHEMINEAU P., 1978. Recherche de l'origine de l'effet dépressif du plasma séminal sur l'aptitude des spermatozoïdes de bouc à supporter la congélation. D. E. A. Univ. P. et M. Curie, Paris VI.

CORTEEL J. M., 1974. Viabilité des spermatozoïdes de bouc conservés et congelés avec ou sans leur plasma séminal : effet du glucose. Ann. Biol. anim. Bioch. Biophys., 14, 741-745.

CORTEEL J. M., 1975. Effet du «lavage » sur la conservation des spermatozoïdes de bouc à basse température. Ann. Biol. anim. Bioch. Biophys., 15, 525-528.

CORTEEL J. M., LEBCEUF B., BARIL G., MARCELLIER N., 1980. In vitro survival and fertilizing ability of dairy goat sperm collected during the breeding season. Proc. IXth int. Congr. Anim. Reprod. artif. Insem., Madrid.

DOTT H. M., 1974. The effects of bovine seminal plasma on the impedance change frequency and glycolysis of bovine epididymal spermatozoa. J. Reprod. Fert., 38, 147-156.

DOTT H. M., HARRISON R. A. P., FOSTER G. C. A., 1979. The mainfenance of motility and the surface properties of epididymal spermatozoa from bull, rabbit and ram in homologous seminal and epididymal plasma. J. Reprod. Fert., 55, 113-124.

ESTEVA-HERNANDEZ A. J., 1976. Effer de la cowperectomie et du lovage sur la congélatibilité des spermafozoïdes de bouc. D. E. A. Univ. P. et M. Curie, Paris VI.

HAAG F. M., 1959. Evaluation of "dismount 》 semen in thoroughbred horse breeding. J. amer. Vet. Med. Assoc., 134, 312.

HALLER O., 1978. Contribution à l'éfude de l'action du plasma séminal sur la conservation « in vitro » des spermatozoìdes de bouc; Mémoire de fin d'études ESIPTA.

HALLER O., CHEMINEAU P., CORTEEL J. M., 1978. Résultats non publiés.

HAMNER C. E., WILLIAMS W. L., 1961. The effect of light on the respiration of spermatozoa. Biochem. biophys. Res. Comm., 5, 316-319.

HARTREE E. F., MANN T., 1960. Phospholipids in mammalian semen. J. Reprod. Fert., 1, 23-29.

IRITANI A., NISHIKAWA Y., 1964. Studies on the egg-yolk coagulating enzyme in goat semen. VI. On the chemical properties of the ejaculated semen and the secretion of accessory sexual organs in the goat. Jap. J. Anim. Reprod., 10, 44-51.

JONES R. C., 1976. The nature of ultrastructural changes induced by exposure of spermatozoa to lysolecithin. Theriogenology, 6, 656.

LASLEY J. K., BOGART R., 1944. A comparative study of epididymal and ejaculated spermatozoa of the boar. J. Anim. Sci., 3, 360-370.

LOCATELLI A., CHEMINEAU P., BARIL G., PISSELET C., DE REVIERS M.-T., CORTEEL J.-M., 1978. L'ablation des glandes de Cowper chez le bouc : approches chirurgicale, histologique et physiologique (résultats non publiés).

MANN T., 1964. The biochemistry of semen and of the male reproductive tract. Methuen and Co., Lond. ; J. Wiley and sons Inc., New York, 493 pp.

MOORE H. D. M., HALL G. A., HIBBITT K. G., 1976. Seminal plasma proteins and the reaction of spermatozoa from intact boars and from boars without seminal vesicles to cooling. J. Reprod. Fert., 47, 39-45. 
MOORE H. D. M., HIBBITT K. G., 1977. Fertility of boar spermatozoa after freezing in the absence of seminal vesicular proteins. J. Reprod. Fert., 50, 349-352.

MULDER E., VAN DEN BERG J. W. O., VAN DEENEN I. I. M., 1965. Metabolism of red cell lipids. II. Conversion of lysophosphoglycerides. Biochem. biophys. Acta, 106, 118-127.

NORMAN C., GOLDBERG E., JOHNSON C. E., PORTERFIELD I. D., 1959. Effect of visible light on motility, life-span and respiration of bovine spermatozoa. J. Dairy Sci., 42, 937 (abstr.).

PAQUIGNON M., MARTINAT-BOTTE F., BARITEAU F., BOSC M. J., COUROT M., MAULEON P., SIGNORET J. P., 1978. Préoccupations et connaissances techniques en matière de reproduction porcine, 63-92. Journées Rech. porcine en France I.T.P. Ed., Paris.

PELOT J., OLLIVIER J. P., TOURNEUR J. C., COUROT M., 1976. Semen quality and fertility in bovine artificial insemination after natural or synchronized estrus. VIllth int. Congr. anim. Reprod. artif. Insem., Cracow, IV, 850-853.

POOLE A. R., HOWELL J. I., LUCY J. A., 1970. Lysolecithin and cell fusion. Nature Lond., 227, 810-813.

PURSEL V. G., JOHNSON L. A., RAMPACEK G. B., 1972. Acrosome morphology of boar spermatozoa incubated before cold shock. J. Anim. Sci., 34, 278-283.

ROY A., 1957. Egg-yolk coagulating enzyme in the semen and Cowper's gland of the goat. Nature, 179, 318-319.

SALISBURY G. W., VAN DEMARK N. L., LODGE J. R., 1978. Physiology of reproduction and artificial insemination of cattle, 798. G. W. SALISBURY Ed., W. H. Freeman and Co., San Francisco, USA.

SHANNON P., 1965. Presence of a heat-labile toxic protein in bovine seminal plasma. J. Dairy Sci., 48, $1362-1365$.

SHANNON P., CURSON R., 1972. Toxic effect and action of dead sperm on diluted bovine semen. J. Dairy Sci., 55, 614-620.

TOSIC J., 1947. Mechanisms of hydrogen peroxide formation by spermatozoa and the role of amino acids in sperm motility. Nature Lond., 159, 544.

TOSIC J., WALTON A., 1946. Respiration of spermatozoa in egg-yolk medium. Nature Lond., 156, 507-508.

TOSIC J., WALTON A, 1960. Metabolism of spermatozoa. The formation and elimination of hydrogen peroxide by spermatozoa and effects on motility and survival. Biochem. J., 47, 199-122.

WERTHESSEN N. T., 1956. In : T. MANN, Male sex hormone and its role in reproduction (Discussion). Rec. Prog. Hormone Res., 12, 370.

WERTHESSEN N. T., MARDEN W., HAAG F., GOLDZIEHER J. W., 1957. The influence of seminal fluid on fertility. Proc. Soc. Study Fertil., 8, 42. 